${ }^{1}$ Área de Investigación en Medicina Interna, Servicio de Clínica Médica. Hospital Italiano de Buenos Aires, Argentina. ${ }^{2}$ Central de Emergencias de Adultos. Hospital Italiano de Buenos Aires, Argentina.

aMagíster en Investigación Clínica.

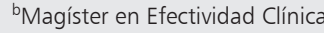
y Sanitaria. 'Enfermero.

${ }^{\mathrm{d} D o c t o r}(\mathrm{PhD})$ en Ciencias de la Salud.

Trabajo no recibió financiamiento. Los autores declaran no tener conflictos de interés.

Recibido el 20 de noviembre de 2019, aceptado el 7 de mayo de 2020.

Correspondencia a: María Florencia Grande Ratti https://orcid.org/0000-0001$8622-8230$

Juan D. Perón 4190 (C1181ACH) Buenos Aires, Argentina. maria.grande@hospitalitaliano. org.ar

\section{Medición del subtriaje como indicador de calidad y seguridad en un servicio de urgencias}

\author{
MARÍA FLORENCIA GRANDE-RATTII,2,a, JORGE ARIEL ESTEBAN²,b, \\ DAMAZO MONGELOS ${ }^{2, c}$, MARIO HERNÁN DÍAZ ${ }^{2}$, \\ DIEGO HERNÁN GIUNTA ${ }^{1, \mathrm{~d}}$, BERNARDO JULIO MARTÍNEZ ${ }^{2}$
}

\section{Undertriage as quality of care parameter in an emergency department}

Background: Undertriage or the underestimation of the urgency of the condition of a person arriving in an emergency department (ED) represents a measure of quality care. Aim: To estimate the prevalence of undertriage in a high complexity hospital of Argentina; to describe characteristics and mortality of these patients. Material and Methods: All consultations admitted to the ED during 2014 were analyzed. Those assigned to a lower level of admission risk (classified as Emergency Severity Index -ESI- 3 to 5) but required hospitalization in intensive care units (ICU) as the first hospitalization place were considered as an undertriage. A random sample of correctly categorized admissions (ESI 1 or 2), who were subsequently hospitalized in the ICU, was selected as a comparison group. Results: The global undertriage prevalence was 0.30\% (316/104,832). Among patients admitted to the ICU, the prevalence was 21\% (316/1,461; 95\% confidence intervals (CI) 19-24). The 316 patients whose severity was underestimated had a median age of 73 years, and admitted between 7 a.m. and 9 p.m. in a greater proportion. Overall hospital mortality was 8.9\% (95\% CI 6.78-11.38), and all deaths occurred after the patient was transferred from the emergency room. There were no differences in mortality between patients with correct triage or undertriage (11 and 7\% respectively, $p=0.09$ ). No differences were observed either in the total number of critical interventions during care in the first 24 hours. Significant differences were observed in requirements for mechanical ventilation (11 and $4 \%$ respectively, $p=0.01$ ), orotracheal intubation (10 and $5 \%$ respectively $p=0.01$ ) and non-invasive ventilation ( 8 and $4 \%$ respectively, $p=0.05$ ). Conclusions: Undertriage rate in this series was low, but it can be improved.

(Rev Med Chile 2020; 148: 602-610)

Keywords: Emergency Medical Services; Epidemiology; Quality of Health Care; Hospital Mortality; Triage.
$\mathrm{E}$ 1 triaje permite identificar a los pacientes con afecciones críticas y sensibles a tiempo, para priorizar su atención por encima de aquellos que pueden esperar ${ }^{1}$, con el fin de brindar la atención necesaria en el lugar y el momento oportuno con los recursos adecuados de manera eficiente ${ }^{2,3}$. Los sistemas que han demostrado validez y repro- ducibilidad clasifican a los pacientes según tres principios: gravedad potencial de su condición clínica, signos vitales y cantidad de recursos que se requerirán ${ }^{4}$.

La infravaloración al ingreso del paciente en los servicios de urgencias (SU), fenómeno conocido como subtriaje ${ }^{5}$, expone al paciente a que su 
condición clínica empeore y representa un factor determinante en demorar el ingreso a unidades de cuidados intensivos (UCI), que está asociado a mayor mortalidad 6 . Su prevalencia varía según las publicaciones entre 14\%, en sistemas implementados durante algún tiempo, y $38 \%$ en sistemas con implementación reciente $e^{5,7-10}$.

Si bien el subtriaje es un fenómeno complejo y sus razones no están completamente definidas, puede empeorar ante el fenómeno de hacinamiento que afecta a los SU a nivel mundial ${ }^{11}$, atentando contra la seguridad del paciente. Se conocen algunos factores asociados o más susceptibles de ser infravalorados como los motivos de consulta inespecíficos, la falta de identificación de situaciones de alto riesgo y la interpretación inadecuada de los signos vitales en pacientes mayores de 65 años $^{12}$.

Este estudio se propuso estimar la prevalencia de subtriaje en nuestro medio, describir las características de estos pacientes y explorar la mortalidad hospitalaria, ya que esta información podría ser relevante para evaluar el desempeño del proceso de clasificación y también implementar cambios que podrían mejorar la calidad de atención y la seguridad del paciente. Como objetivo secundario, se exploran los factores asociados al subtriaje.

\section{Material y Métodos}

Se realizó un estudio de corte transversal en el Hospital Italiano de Buenos Aires (HIBA) de Argentina, que cuenta con 750 camas, 200 de ellas correspondientes a Unidad de Cuidados Intensivos (UCI): Unidad Coronaria (UCO), Unidad de Cuidados Intermedios (UTIM) y Unidad de Cuidados Intensivos Adulto (UTIA). También cuenta con una prepaga médica institucional denominada Plan de Salud (PS), un seguro médico que brinda servicios por una tarifa mensual.

$\mathrm{Al}$ ingreso, el proceso de triaje define el área de atención asignada y la categoría de triaje para su prioridad de atención. El mismo es una adaptación local de la escala ESI (5-level Emergency Triage Severity Index $)^{13}$ realizada por personal de enfermería entrenado ${ }^{14}$. Este mecanismo permite identificar los pacientes críticos, con condiciones inestables que amenazan (ESI nivel 1) o están en riesgo de amenazar (ESI nivel 2) la vida, lo que condiciona una respuesta asistencial inmediata o no demorable, respectivamente. Si no es así, el paciente pasa a ser categorizado en los restantes niveles (niveles ESI 3 a 5) de acuerdo a la estimación de recursos que el paciente va a precisar en su asistencia. Durante su estancia en SU, los pacientes son reevaluados periódicamente.

Se incluyeron la totalidad de pacientes que ingresaron en SU entre enero y diciembre de 2014 como denominador, en contexto de la creación de un tablero de control, herramienta administrativa aplicable a cualquier organización cuyo objetivo y utilidad básica es diagnosticar adecuadamente una situación. Se definió subtriaje a aquellos pacientes que fueron asignados a niveles de ESI 3 a 5 al ingreso en urgencias, pero que al cierre de la epicrisis requirieron hospitalización en la UCI como primer área, esto excluye las transferencias posteriores a estas áreas durante la hospitalización (aquellos que ingresaron a áreas de internación general y se complicaron).

Se decidió, desde el inicio del proyecto (no post hoc), incluir un grupo control con una muestra aleatoria de pacientes con correcta clasificación, con una relación de 1:1, definidos como aquellos categorizados con niveles de ESI 1-2 y que también requirieron hospitalización en UCI como primer área de ingreso. Esta decisión radica en permitir explorar factores asociados al subtriaje, para lo cual se requiere un grupo comparador.

Los datos fueron obtenidos desde bases administrativas secundarias de alta calidad, correspondientes al sistema de información de atención: historia clínica electrónica (HCE). Para explorar el consumo de recursos se utilizó la herramienta Life Saving Interventions, que permitió explorar las intervenciones críticas requeridas durante las primeras $24 \mathrm{~h}$.

Las variables cuantitativas se presentaron como media y desviación estándar o mediana y rango intercuartilo según la distribución observada y las variables categóricas como frecuencia absoluta y proporciones, con sus respectivos intervalos de confianza de 95\%. Para las comparaciones se utilizó chi-cuadrado o Fisher y T-Test o Mann-Whitney. Las probabilidades inferiores al $5 \%$ se consideraron significativas. Utilizamos STATA13.

La tasa de subtriaje global se calculó como la proporción de pacientes con calificación infravalorada al ingreso sobre la totalidad de los que ingresaron al SU en el período de estudio. La tasa 
de subtriaje en UCI se calculó como la proporción en relación al número total de hospitalizaciones no programadas en UCI.

Este estudio fue aprobado por el comité de ética institucional, y no requirió consentimiento informado.

\section{Resultados}

Durante el período de estudio se detectaron 316 casos de subtriaje; mientras que 1.145 se categorizaron correctamente. De estos últimos, se seleccionó una muestra aleatoria. Solo 2 pacientes fueron excluidos debido al diagnóstico (Figura 1).

La prevalencia global de subtriaje resultó $0,30 \%(316 / 104832)$ con IC $95 \% 0,26$ a $0,33 \%$. La prevalencia de subtriaje en los pacientes ingresados en UCI resultó $21 \%(316 / 1461)$ con IC 95\% 19-24\%.

Las características basales de los pacientes se describen en la Tabla 1. Los pacientes con subtriaje tuvieron mayor proporción de ingreso $(46,67 \%$ (147/315]) entre las 7 am y las 9 pm, y en los primeros 3 meses del año $(42,22 \%(133 / 315])$. La admisión en UCO representó el 78,10\% (246/315) y la edad media fue de 73 años (RIC 21), con una proporción de pacientes afiliados a PS de $50 \%$.

En comparación con el grupo control, la proporción de pacientes que ingresaron deambulando por sus propios medios fue mayor en el grupo subtriaje ( $90 \%$ versus $60 \%$, respectivamente, $\mathrm{p}<0,01)$, y hubo diferencias significativas en el tiempo de demora para la atención médica (definido como tiempo transcurrido entre la llegada del paciente al SU hasta que el médico lo atendió efectivamente) con mediana de 24,69 $\mathrm{min}$ en grupo subtriaje frente a $12,35 \mathrm{~min}$ en grupo de clasificación correcta $(\mathrm{p}<0,01)$. Consistentemente, también hubo una diferencia significativa en el tiempo de atención médica en SU (definido como tiempo transcurrido entre la asistencia efectiva por médico en SU hasta el trámite administrativo de hospitalización): mediana de 175 min en grupo subtriaje versus 102 min en grupo con clasificación correcta $(\mathrm{p}<0,01)$. No hubo diferencias estadísticas en relación al sexo del paciente, el día de llegada al SU, el turno de triaje, o las comorbilidades preexistentes según índice de Charlson.

La tasa de mortalidad intrahospitalaria global fue 8,88\% (IC 95\% 6,78-11,38), los 56 fallecimientos ocurrieron durante la hospitalización, resultando nula la mortalidad específica en el SU. La Tabla 2 presenta las variables inherentes a la hospitalización y del seguimiento. No hubo diferencias significativas con respecto a la mortalidad hospitalaria, ocurriendo 6,98\% de subtriaje versus $10,79 \%$ en grupo control $(p=0,09)$. Si bien tampoco hubo diferencias significativas con el número total de intervenciones críticas, detectamos que el porcentaje de necesidad de ventilación mecánica $(10,79 \%$ versus $4,44 \% ; \mathrm{p}=0,01)$, intubación

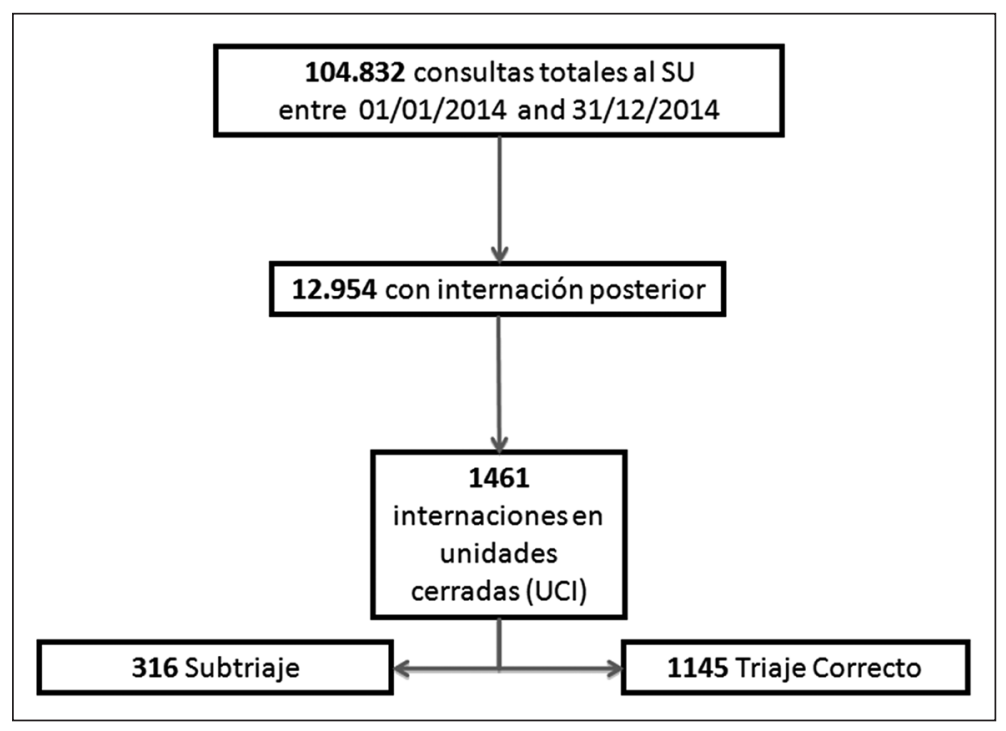

Figura 1. Flujograma de pacientes incluidos. 
Tabla 1. Características basales de la población incluida

\begin{tabular}{|c|c|c|c|}
\hline & $\begin{array}{c}\text { Triaje correcto } \\
\text { n: } \mathbf{3 1 5}\end{array}$ & $\begin{array}{l}\text { Subtriaje } \\
\text { n: } \mathbf{3 1 5}\end{array}$ & p valor \\
\hline Edad, en años* & $76(20)$ & $73(21)$ & 0,004 \\
\hline Sexo masculino & $54,60 \%(172)$ & $59,87 \%(187)$ & 0,227 \\
\hline Tipo de cobertura médica & & & 0,027 \\
\hline Plan de salud & $60 \%(189)$ & $50,16 \%(158)$ & \\
\hline PAMI / IOMA** & $12,70 \%(40)$ & $13,02 \%(41)$ & \\
\hline Otra & $27,30 \%(86)$ & $36,83 \%(116)$ & \\
\hline Mes & & & 0,055 \\
\hline Enero & $12,38 \%(39)$ & $15,87 \%(50)$ & \\
\hline Febrero & $8,89 \%(28)$ & $14,60 \%(46)$ & \\
\hline Marzo & $6,35 \%(20)$ & $11,75 \%(37)$ & \\
\hline Abril & $7,94 \%(25)$ & $6,67 \%(21)$ & \\
\hline Mayo & $8,57 \%(27)$ & $7,62 \%(24)$ & \\
\hline Junio & $10,79 \%(34)$ & $8,25 \%(26)$ & \\
\hline Julio & $8,25 \%(26)$ & $8,25 \%(26)$ & \\
\hline Agosto & $4,76 \%(15)$ & $4,44 \%(14)$ & \\
\hline Septiembre & $6,35 \%(20)$ & $5,40 \%(17)$ & \\
\hline Octubre & $7,94 \%(25)$ & $5,08 \%(16)$ & \\
\hline Noviembre & $6,35 \%(20)$ & $5,71 \%(18)$ & \\
\hline Diciembre & $11,43 \%(26)$ & $6,35 \%(20)$ & \\
\hline Día de ingreso & & & 0,224 \\
\hline Laborable & $67,62 \%(213)$ & $72,06 \%(227)$ & \\
\hline No laborable & $32,38 \%(102)$ & $27,94 \% \quad(88)$ & \\
\hline Turno de triaje & & & 0,057 \\
\hline 7-14 h (mañana) & $36,19 \%(114)$ & $37,14 \%(117)$ & \\
\hline $14-21 \mathrm{~h}$ (tarde) & $40,32 \%(127)$ & $46,67 \%(147)$ & \\
\hline 21-7 h (noche) & $23,49 \% \quad(74)$ & $16,19 \% \quad(51)$ & \\
\hline Tipo de ingreso & & & $<0,001$ \\
\hline En ambulancia & $59,36 \%(187)$ & $8,25 \% \quad(26)$ & \\
\hline En silla & $2,86 \% \quad(9)$ & $1,27 \% \quad(4)$ & \\
\hline Caminando & $37,78 \%(119)$ & $90,48 \%(285)$ & \\
\hline Índice de Charlson, categórica & & & 0,793 \\
\hline Sin comorbilidad & $66,03 \%(208)$ & $60,63 \%(191)$ & \\
\hline Comorbilidad baja & $13,33 \% \quad(42)$ & $16,50 \% \quad(52)$ & \\
\hline Comorbilidad alta & $20,64 \% \quad(65)$ & $22,85 \% \quad(72)$ & \\
\hline Tiempo de demora, en minutos* & $12,35(\operatorname{RIC} 16,6)$ & 24,69 (RIC 43,23) & $<0,001$ \\
\hline Tiempo de atención en CEA, en minutos* & $102,15(\operatorname{RIC} 165,6)$ & 175,68 (RIC 256,98) & $<0,001$ \\
\hline
\end{tabular}

*Mediana (RIC: rango intercuartil). **PAMI: programa de atención médica integral y IOMA: obra social de la provincia de Buenos Aires. 
Tabla 2. Variables relacionadas a la hospitalización en UCI

\begin{tabular}{|c|c|c|c|}
\hline & $\begin{array}{c}\text { Triaje correcto } \\
\text { n: } 315\end{array}$ & $\begin{array}{l}\text { Subtriaje } \\
\text { n: } 315\end{array}$ & p valor \\
\hline Tipo de Unidad de Cuidados Intensivos (UCI) & & & 0,031 \\
\hline Unidad coronaria & $72,70 \%(229)$ & $78,10 \%(246)$ & \\
\hline Terapia intensiva & $13,33 \% \quad(42)$ & $6,98 \% \quad(22)$ & \\
\hline Terapia intermedia & $13,97 \% \quad(44)$ & $14,92 \% \quad(47)$ & \\
\hline \multicolumn{4}{|l|}{ Recursos intensivos en primeras $24 \mathrm{~h}$} \\
\hline $\mathrm{ARM}^{*}$ & $10,79 \% \quad(34)$ & $4,44 \% \quad(14)$ & 0,003 \\
\hline $10 T^{+}$ & $10,16 \% \quad(32)$ & $4,76 \% \quad(15)$ & 0,010 \\
\hline VNI\# & $8,25 \% \quad(26)$ & $4,44 \% \quad(14)$ & 0,050 \\
\hline Vía aérea quirúrgica & -- & -- & -- \\
\hline Desfibrilación & $0,95 \% \quad(3)$ & $1,27 \% \quad(4)$ & 1 \\
\hline Cardioversión & $0,95 \% \quad$ (3) & $1,59 \% \quad(5)$ & 0,725 \\
\hline Marcapasos externo & $1,27 \% \quad(4)$ & $1,90 \% \quad(6)$ & 0,752 \\
\hline Pericardiocentesis & -- & $1,27 \% \quad(4)$ & 0,124 \\
\hline Tubo de tórax & $0,32 \% \quad(1)$ & $0,63 \% \quad(2)$ & 1 \\
\hline Toracotomía & -- & -- & -- \\
\hline Vía intraósea & -- & -- & -- \\
\hline Monitoreo hemodinámico & $87,94 \%(277)$ & $86,35 \%(272)$ & 0,552 \\
\hline Reanimación con volumen & $6,67 \% \quad(21)$ & $9,52 \% \quad(30)$ & 0,189 \\
\hline Sangrado mayor & $7,94 \% \quad(25)$ & $10,48 \%$ & 0,270 \\
\hline Uso de drogas inotrópicas & $8,57 \% \quad(27)$ & $6,67 \% \quad(21)$ & 0,368 \\
\hline Muerte intrahospitalaria & $10,79 \% \quad(34)$ & $6,98 \% \quad(22)$ & 0,093 \\
\hline
\end{tabular}

*ARM: Asistencia respiratoria mecánica. ${ }^{+}$IOT: Intubación orotraqueal. \#VNI: Ventilación no invasiva.

orotraqueal $(10,16 \%$ versus $4,76 \% ; \mathrm{p}=0,01) \mathrm{y}$ la ventilación no invasiva $(8,25 \%$ versus $4,44 \%$; $\mathrm{p}=0,05)$ fueron mayores en el grupo de correcta clasificación, con significancia estadística.

Dado que la mayoría del grupo subtriaje fue admitido en la unidad coronaria (246/315), se decidió realizar un análisis de subgrupo post hoc, excluyéndose estos pacientes, con el fin de reevaluar las características de los pacientes subcategorizados (Tabla 3). Los pacientes del grupo subtriaje que se internaron en terapia fueron mayoritariamente durante la semana y en días laborales de lunes a viernes $(\mathrm{p}=0,02)$, ingresando caminando por sus propios medios $(\mathrm{p}=0,01)$ y la mayoría se trasladó a terapia intermedia.

Se decidió explorar el motivo de ingreso de estos pacientes de acuerdo al diagnóstico registra- do al cierre de epicrisis del episodio de SU (Tabla 4). Los motivos más frecuentes fueron: $26,66 \%$ enfermedad coronaria, $17,46 \%$ insuficiencia cardíaca y 10,16\% arritmias. Siguiendo el análisis de subgrupo post hoc, los motivos de consulta, excluyendo aquellos que ingresaron a UCO, resultaron: $17,39 \%$ enfermedad vascular periférica (aneurisma aorta abdominal), 15,94\% enfermedad cerebrovascular (ACV, hematoma subdural, hemorragia subaracnoidea), 15,94\% insuficiencia cardíaca y $14,49 \%$ hemorragia digestiva.

\section{Discusión}

La prevalencia de subtriaje global resultó similiar a la reportada en la bibliografía ${ }^{5,7-10}$. Si bien este fenómeno no resultó frecuente en nuestro medio, 
Tabla 3. Análisis de subgrupo (excluyendo unidad coronaria)

\begin{tabular}{|c|c|c|c|}
\hline & $\begin{array}{c}\text { Triaje correcto } \\
\text { n: } 86\end{array}$ & $\begin{array}{c}\text { Subtriaje } \\
\text { n: } 69\end{array}$ & p valor \\
\hline Sexo masculino & $47,67 \%(41)$ & $50,72 \%(35)$ & 0,706 \\
\hline Edad, en años* & 70,5 (RIC 30) & 65 (RIC 34) & 0,293 \\
\hline Tipo de cobertura médica & & & 0,881 \\
\hline Plan de salud & $45,35 \%(39)$ & $47,83 \%(33)$ & \\
\hline PAMI / IOMA** & $11,63 \%(10)$ & $13,04 \%(9)$ & \\
\hline Otra & $43,02 \%(37)$ & $39,13 \%(27)$ & \\
\hline Mes & & & 0,750 \\
\hline Enero & $17,44 \%(15)$ & $23,19 \%(16)$ & \\
\hline Febrero & $12,79 \%(11)$ & $20,29 \%(14)$ & \\
\hline Marzo & $4,65 \% \quad(4)$ & $7,25 \% \quad(5)$ & \\
\hline Abril & $6,98 \% \quad(6)$ & $2,90 \% \quad(2)$ & \\
\hline Mayo & $5,81 \% \quad(5)$ & $8,70 \% \quad(6)$ & \\
\hline Junio & $9,30 \% \quad(8)$ & $5,80 \% \quad(4)$ & \\
\hline Julio & $8,14 \% \quad(7)$ & $4,35 \% \quad$ (3) & \\
\hline Agosto & $4,65 \% \quad(4)$ & $4,35 \% \quad(3)$ & \\
\hline Septiembre & $5,81 \% \quad(5)$ & $5,80 \% \quad(4)$ & \\
\hline Octubre & $10,47 \%$ & $4,35 \% \quad(3)$ & \\
\hline Noviembre & $5,81 \% \quad(5)$ & $4,35 \% \quad(3)$ & \\
\hline Diciembre & $8,14 \% \quad(7)$ & $8,70 \% \quad(6)$ & \\
\hline Día de ingreso laborable & $52,33 \%(45)$ & $69,57 \%(48)$ & 0,029 \\
\hline Turno de triaje & & & 0,207 \\
\hline 7-14 h (mañana) & $40,70 \%(35)$ & $34,78 \%(24)$ & \\
\hline 14-21 h (tarde) & $37,21 \%(32)$ & $50,72 \%(35)$ & \\
\hline 21-7 h (noche) & $22,09 \%(19)$ & $14,49 \%(10)$ & \\
\hline Índice de Charlson, categórica & & & 0,876 \\
\hline Sin comorbilidad & $36,05 \%(31)$ & $43,48 \%(30)$ & \\
\hline Comorbilidad baja & $17,44 \%(15)$ & $21,74 \%(15)$ & \\
\hline Comorbilidad alta & $23,26 \%(20)$ & $23,19 \%(16)$ & \\
\hline Tipo de ingreso & & & $<0,001$ \\
\hline En ambulancia & $63,95 \%(55)$ & $13,04 \% \quad(9)$ & \\
\hline En silla & $1,16 \%$ & $1,44 \% \quad(1)$ & \\
\hline Caminando & $34,89 \%(30)$ & $85,52 \%(59)$ & \\
\hline Tipo de UCI & & & 0,033 \\
\hline Terapia intermedia & $51,16 \%(44)$ & $68,12 \%(47)$ & \\
\hline Terapia intensiva & $48,84 \%(42)$ & $31,88 \%(22)$ & \\
\hline Mortalidad intrahospitalaria & $16,28 \%(14)$ & $10,14 \%$ & 0,267 \\
\hline Número de intervenciones críticas* & $1(\mathrm{RIC} 3)$ & 1 (RIC 3) & 0,785 \\
\hline
\end{tabular}

*Mediana (RIC: rango intercuartil). **PAMI: programa de atención médica integral y IOMA: obra social de la provincia de Buenos Aires. 
Tabla 4. Motivo de ingreso a CEA, según subgrupo

\begin{tabular}{|c|c|c|c|}
\hline & $\begin{array}{c}\text { Triaje correcto } \\
\text { n: } 315\end{array}$ & $\begin{array}{c}\text { Subtriaje } \\
\text { n: } \mathbf{3 1 5}\end{array}$ & p valor \\
\hline Arritmias & $11,43 \%(36)$ & $10,16 \%(32)$ & 0,608 \\
\hline Enfermedad cardiovascular & $31,42 \%(99)$ & $26,66 \%(84)$ & 0,188 \\
\hline Enfermedad vascular periférica & $1,26 \% \quad(4)$ & $1,26 \% \quad(4)$ & 1 \\
\hline Enfermedad tromboembólica & $1,58 \% \quad(5)$ & $3,81 \%(12)$ & 0,138 \\
\hline Enfermedad cerebrovascular & $10,47 \%(33)$ & $8,25 \%(26)$ & 0,338 \\
\hline Hemorragia digestiva & $2,53 \% \quad(8)$ & $4,44 \%(14)$ & 0,193 \\
\hline Insuficiencia cardíaca & $18,09 \%(57)$ & $17,46 \%(55)$ & 0,835 \\
\hline Patología pleuropericárdica & $5,08 \%(16)$ & $7,62 \%(24)$ & 0,191 \\
\hline Renal & -- & $2,85 \% \quad(9)$ & 0,004 \\
\hline Infecciosas & $7,30 \%(23)$ & $6,66 \%(21)$ & 0,755 \\
\hline Oncológicas & $0,63 \% \quad(2)$ & $1,58 \% \quad(5)$ & 0,451 \\
\hline Quirúrgicas & $1,26 \% \quad(4)$ & $1,26 \% \quad(4)$ & 1 \\
\hline Metabólicas & $1,26 \% \quad(4)$ & $0,95 \% \quad(3)$ & 1 \\
\hline Otras & $7,62 \%(24)$ & $6,98 \%(22)$ & 0,759 \\
\hline
\end{tabular}

permite reflexionar e implementar medidas para mantener o incluso mejorar este indicador de gestión ${ }^{15}$.

Los pacientes infravalorados fueron en mayor proporción entre las 7 am y 9 pm, probablemente relacionado con el mayor flujo natural de pacientes en el SU, pero $42,22 \%$ ocurrió entre enero y marzo, que podría deberse a las vacaciones, coincidiendo con atención por parte de profesionales con menor experiencia (reemplazos o residentes).

El 78,10\% de subtriaje fue admitido posteriormente en UCO. Desde el punto de vista gerencial, nos interesó profundizar los motivos de consulta principales de este subgrupo. Dado que se trató de situaciones clínicas frecuentes en SU, esta información nos permitió implementar un mecanismo de mejora mediante la creación de un circuito estructurado para la atención de estos pacientes (Unidad de Dolor Torácico), que permite a los especialistas en cardiología estar avisados en forma temprana sobre la existencia de estos pacientes.

En contraste con la bibliografía ${ }^{16}$, nuestro estudio arrojó que el hecho de ser joven y no afiliado a prepaga institucional resultaron factores de riesgo para infravalorar al paciente (p 0,01). Quizás, el joven es fácilmente subestimado o el adulto mayor se ve favorecido debido a su funcionalidad disminuida que lo inhabilita para esperar sentado, otorgándole cierta prioridad. Los pacientes de otros prestadores podrían ser infravalorados por no estar disponible los antecedentes en la HCE.

Como principal limitación, nuestra definición fue pragmática y basada en la factibilidad de medición más allá de las definiciones bibliográficas de subtriaje $^{17}$. Sin embargo, no permite discriminar el desacuerdo entre la evaluación inicial por parte de enfermería del triaje y la evaluación final por parte de un médico (decisión de hospitalización en UCI), si el mismo fue realmente una evaluación errónea en la interpretación del paciente a su ingreso ${ }^{18} \mathrm{o}$ fue explicado debido a la evolución tórpida durante el proceso de atención. Se intentó explorar esto mediante un enfoque simplista. Los motivos de consulta más frecuentes en los casos de subtriaje fueron: $17,39 \%$ de enfermedad vascular periférica, $15,94 \%$ de enfermedad cerebrovascular y 14,49\% de hemorragia digestiva, datos de gran importancia desde el punto de vista gerencial. 
Creemos que la herramienta de intervenciones críticas utilizada como aproximación de recursos de salud podría no haber sido la ideal, porque no tiene en consideración otras intervenciones críticas que se utilizaron durante la estadía del paciente crítico en el SU como: administración de medicación endovenosa que requiere monitoreo cardíaco (por ejemplo: agentes trombolíticos, digoxina) o la realización de estudios complementarios como cinecoronariografía; $u$ otros procedimientos habituales (colocación de sonda nasogástrica, accesos arteriales, o videoendoscopía digestiva alta).

La mortalidad hospitalaria se exploró como una aproximación de las consecuencias críticas del subtriaje y eventual infratratamiento consecuente, no encontrándose diferencias estadísticamente significativas. Podría ser interesante para futuras investigaciones un análisis en profundidad de los fallecidos, que explore las causas y detecte potenciales errores clínicos como lecciones aprendidas.

Adicionalmente, cabe mencionar que la inclusión de elementos mediados por la hospitalización escapan del alcance del triaje en la primera atención al ingreso. Los resultados de las maniobras diagnósticas, terapéuticas y de seguimiento en la atención cerrada no necesariamente guardan relación con la priorización del triaje y habitualmente son, además, equipos tratantes diferentes.

Como una gran fortaleza, representa una primera aproximación en nuestro país y es un disparador potencial para el circuito de mejora. El subtriaje sigue siendo un tema de calidad importante en cualquier sistema de salud ${ }^{19}$.

En conclusión, la prevalencia de subtriaje resultó baja como indicador de calidad, sin encontrarse diferencias significativas en la mortalidad intrahospitalaria en comparación con el grupo de triaje correcto. Los factores asociados al subtriaje resultaron: edad, modo de ingreso y tipo de unidad de cuidados críticos que admite el paciente. No es un dato menor que los pacientes jóvenes, que ingresan deambulando por sus medios y que consultan por motivos de consulta cardiológicos, se suelen subestimar con mayor frecuencia. Esto permitiría contemplar estas banderas rojas (señales de advertencia) para actuar a tiempo, que permita identificar en forma temprana este perfil epidemiológico de paciente para valorarlo cuidadosamente. Estos datos nos permitieron generar la medición sistemática y prospectiva de este indicador de calidad y seguridad en la SU, que permite el monitoreo de los procesos con los profesionales involucrados en la atención a modo de lecciones aprendidas. En efecto, el SU cuenta actualmente con este indicador de calidad medido en forma periódica y sistemática, manteniéndose estable su medición a través del tiempo, apoyándonos en las nuevas tecnologías informáticas y las bases secundarias desde registros de HCE.

Agradecimientos: Los autores quisieran agradecer su colaboración a Dr. Javier Pollán como jefe del Servicio de Clínica Médica por el apoyo institucional.

\section{Referencias}

1. Iserson KV, Moskop JC. Triage in medicine, part I: Concept, history, and types. Ann Emerg Med 2007; 49: 275-81.

2. Savatmongkorngul S, Yuksen C, Suwattanasilp C, Sawanyawisuth K, Sittichanbuncha Y. Is a mobile emergency severity index (ESI) triage better than the paper ESI? Intern Emerg Med 2016; 12: 1273-7.

3. Sabour S. Emergency Severity Index (ESI) triage; mobile or paper? Methodological issue on validity and reliability analysis. Intern Emerg Med 2017; 12: 271-2.

4. Soler W, Gómez Muñoz M, Bragulat E, Álvarez A. El triaje: herramienta fundamental en urgencias y emergencias. An Sist Sanit Navar 2010; 33: 55-68.

5. Grossmann FF E al. At risk of undertriage? Testing the performance and accuracy of the emergency severity index in older emergency department patients. Ann Emerg Med. 2012; 60 (3): 317-25.e3. doi: 10.1016/j. annemergmed.2011.12.013. Epub 2012 Mar 7.

6. Yurkova I, Wolf L. Under-triage as a significant factor affecting transfer time between the emergency department and the intensive care unit. J Emerg Nurs. 2011; 37 (5): 491-6. doi: 10.1016/j.jen.2011.01.016. Epub 2011 May 5.

7. Grossmann FF E al. Undertriage in older emergency department patients--tilting against windmills? PLoS One. 2014; 9 (8): e106203. doi: 10.1371/journal. pone.0106203. eCollection 2014.

8. Bergs J E al. Evaluating implementation of the emergency severity index in a Belgian hospital. Bergs J, et al. J Emerg Nurs. 2014.

9. Twomey M E al. Evaluating the construct of triage acuity against a set of reference vignettes developed via modified Delphi method. Emerg Med J. 2014; 31 (7): 562-6. 
doi: 10.1136/emermed-2013-202352. Epub 2013 Apr 24.

10. Storm-Versloot MN E al. Comparison of an informally structured triage system, the emergency severity index, and the manchester triage system to distinguish patient priority in the emergency department. Acad Emerg Med. 2011; 18 (8): 822-9. doi: 10.1111/j.15532712.2011.01122.x.

11. Giunta DH, Pedretti AS, Elizondo CM, Grande Ratti MF, González Bernaldo de Quiros F, Waisman GD, et al. [Analysis of Crowding in an Adult Emergency Department of a tertiary university hospital]. Rev Med Chile 2017; 145: 557-63.

12. Gasperini B, Cherubini A, Fazi A, Maracchini G, Prospero E. Older adults in Emergency Departments: the challenge of undertriage. Intern Emerg Med 2016; 11: 1145-7.

13. de Gracia Adánez Martínez Bartolomé García Pérez Daniel Nova López José Luis Díaz Agea THRCLCM. Evidencias de validez del sistema de triaje Emergency Severity Index en un servicio de urgencias de un hospital general. Emergencias 2015; 27: 301-6.

14. Martínez-Segura E, Lleixà-Fortuño $\mathrm{M}$, Salvadó-Usach
T, Solà-Miravete E, Adell-Lleixà M, Chanovas-Borrás $\mathrm{MR}$, et al. [Competence of triage nurses in hospital emergency departments]. Emergencias 2017; 29: 173-7.

15. Hitchcock M, Gillespie B, Crilly J, Chaboyer W. Triage: an investigation of the process and potential vulnerabilities. J Adv Nurs 2014; 70: 1532-41.

16. Grossmann FF, Zumbrunn T, Frauchiger A, Delport K, Bingisser R, Nickel CH. At risk of undertriage? Testing the performance and accuracy of the emergency severity index in older emergency department patients. Ann Emerg Med 2012; 60: 317-25.e3.

17. Lentz BA, Jenson A, Hinson JS, Levin S, Cabral S, George $\mathrm{K}$, et al. Validity of ED: Addressing heterogeneous definitions of over-triage and under-triage. Am J Emerg Med 2017; 35: 1023-5.

18. Malmström T, Harjola V-P, Torkki P, Kumpulainen S, Malmström R. Triage quality control is missing tools-a new observation technique for ED quality improvement. Int J Qual Health Care 2017; 29: 295-300.

19. Brosinski CM, Riddell AJ, Valdez S. Improving Triage Accuracy: A Staff Development Approach. Clin Nurse Spec 2017; 31: 145-8. 